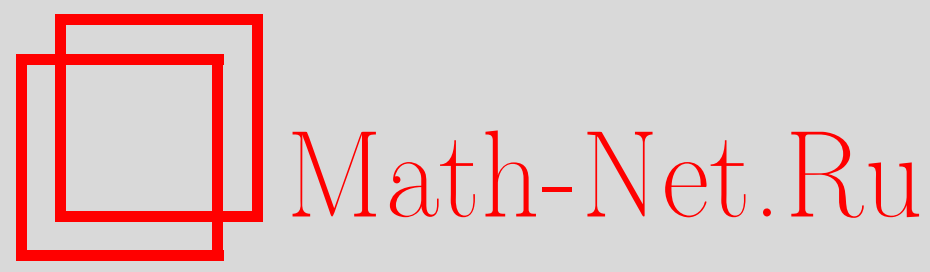

Е. К. Алексеев, О некоторых алгебраических и комбинаторных свойствах корреляционно-иммунных булевых функций, Дискрет. матем., 2010, том 22, выпуск 3, 110-126

DOI: https://doi.org/10.4213/dm1111

Использование Общероссийского математического портала Math-Net.Ru подразумевает, что вы прочитали и согласны с пользовательским соглашением http://www . mathnet.ru/rus/agreement

Параметры загрузки:

IP: 34.227 .88 .159

26 апреля 2023 г., $15: 25: 38$ 


\title{
О некоторых алгебраических и комбинаторных свойствах корреляционно-иммунных булевых функций
}

\author{
(c) 2010 г. $\quad$ Е. К. Алексеев
}

\begin{abstract}
Работа посвящена исследованию свойств корреляционно-иммунных и устойчивых функций в целом. Описание этих свойств проводится с помощью алгебраических объектов и операций.
\end{abstract}

\section{1. Введение}

Использование булевой функции в качестве математической модели криптографического примитива обуславливает наличие у этой функции определенных свойств. Способность противостоять корреляционной атаке отражает понятие корреляционной иммунности булевой функции [7]. Отвлекаясь от нюансов, можно сказать, что это свойство означает отсутствие какой-либо информации в известном значении булевой функции о значениях некоторого подмножества ее аргументов или о значениях некоторых функций от ее аргументов.

Корреляционно-иммунные и устойчивые функции являются (под разными названиями) известными объектами в таких разделах математики как комбинаторика, математическая статистика, теория кодирования и др. (см. [1, 2]). Основное свойство этих функций сделало их широко используемым криптографическим примитивом при построении потоковых и блочных шифров.

Выбор булевой функции для криптографических приложений является сложной оптимизационной задачей относительно параметров, описывающих те или иные криптографические свойства этой функции. Поэтому основные исследования были направлены на выявление связей корреляционной иммунности и устойчивости с другими свойствами булевых функций (см., например, $[5,6,9])$. Множество работ было посвящено также развитию методов синтеза булевых функций, обладающих заданными криптографическими свойствами (см., например. [5, 6, 10, 11]).

Вместе с тем представляет определенный теоретический и практический интерес исследование алгебраических и комбинаторных свойств естественных подмножеств множества корреляционно-иммунных функций в целом. Так как известные характеризации корреляционной иммунности носят теоретико-информационный, спектральный и метрический характер, указанные выше исследования представляются достаточно сложными. Это показывает также относительная малочисленность результатов этого направления. В качестве примера можно привести работу [3]. 
Настоящая работа посвящена исследованию свойств множества корреляционно-иммунных функций в целом, описываемых с помощью алгебраических объектов и операций.

\section{2. Основные определения и понятия}

Пусть $F_{q}$ - конечное поле, состоящее из $q$ элементов, где $q=p^{n}, p-$ простое число. Пусть $V_{n}=F_{2} \times \ldots \times F_{2}-$ векторное пространство наборов длины $n$ с компонентами из $F_{2}$.

Определение 1. Булевой функцией от $n$ переменных называется отображение из $V_{n}$ в $F_{2}$. Множество всех булевых функций от $n$ переменных будем обозначать $\mathscr{F}_{n}$.

В дальнейшем будем придерживаться следующих обозначений: $x_{i}-i$-й вектор из некоторой совокупности векторов, $x^{(j)}-j$-я компонента вектора $x$. Если $n-$ натуральное число, а $c \in\{0,1\}$, то через $c^{n}$ будем обозначать вектор длины $n$, все компоненты которого равны $c$. Константные булевы функции будем обозначать через $\overline{0}$ и $\overline{1}$.

Пусть $L-$ подмножество пространства $V_{n}$. Тот факт, что $L$ является собственным подпространством пространства $V_{n}$, будем обозначать так: $L<V_{n}$.

Плоскостями в $V_{n}$ будем называть смежные классы по подпространствам этого пространства.

Определение 2. Подфункцией булевой функции $f$ называется функция $f^{\prime}$, полученная подстановкой в $f$ констант 0 или 1 вместо некоторых переменных.

Если мы подставляем в функцию $f$ константы $\sigma^{(1)}, \ldots, \sigma^{(s)}$, где $s \leqslant n$, вместо переменных $x^{\left(i_{1}\right)}, \ldots, x^{\left(i_{s}\right)}$ соответственно, то полученная подфункция обозначается $f_{i_{1}, \ldots, i_{s}}^{\sigma^{(1)}, \ldots, \sigma^{(s)}}$.

Определение 3. Алгебраической нормальной формой (АНФ) функции $f$ из $\mathscr{F}_{n}$ называется ее представление (однозначное) в виде полинома из кольца $F_{2}\left[x^{(1)}, \ldots, x^{(n)}\right]$, степень которого по каждой переменной не превосходит 1.

Определение 4. Носителем функции $f \in \mathscr{F}_{n}$ называется множество

$$
1_{f}=\left\{x \in V_{n} \mid f(x)=1\right\} .
$$

Определение 5. Скалярным произведением векторов $x, u \in V_{n}$ называется сумма

$$
\langle x, u\rangle=\bigoplus_{i=1}^{n} x^{(i)} u^{(i)} .
$$

Определение 6. Весом $\operatorname{wt}(f)$ булевой функции $f \in \mathscr{F}_{n}$ называется мощность ее носителя $1_{f}$.

Определение 7. Преобразованием Уолша-Адамара булевой функции $f$ называется целочисленная функция на $V_{n}$, определяемая равенством

$$
W_{f}(u)=\sum_{x \in V_{n}}(-1)^{f(x) \oplus\langle x, u\rangle},
$$

где суммирование производится в действительной области. Для каждого $u \in V_{n}$ значение $W_{f}(u)$ называется коэффициентом Уолша-Адамара. 
Определение 8. Преобразованием Фурье булевой функции $f \in \mathscr{F}_{n}$ называется целочисленная функция на $V_{n}$, определяемая равенством

$$
F_{f}(u)=\sum_{x \in V_{n}} f(x)(-1)^{\langle x, u\rangle},
$$

где суммирование производится в действительной области. Для каждого $u \in V_{n}$ значение $F_{f}(u)$ называется коэффициентом Фурье.

Теорема 1 ([1]). Коэффициенты Фурье и коэффициенты Уолиа-Адамара связань соотношением

$$
W_{f}(u)=2^{n} \delta(u)-2 F_{f}(u),
$$

где $\delta(0)=1 u \delta(u)=0$ при $u \neq 0$.

Определение 9. Булева функция $f\left(x^{(1)}, x^{(2)}, \ldots, x^{(n)}\right) \in \mathscr{F}_{n}$ называется корреляционноиммунной порядка $m, 0<m \leqslant n$, если для любых $1 \leqslant i_{1}<i_{2}<\ldots<i_{m} \leqslant n, a^{(i)} \in F_{2}$, $j=1, \ldots, m$, выполняются соотношения

$$
\operatorname{wt}\left(f_{i_{1}, \ldots, i_{m}}^{a^{(1)}, \ldots, a^{(m)}}\right)=\frac{\mathrm{wt}(f)}{2^{m}} .
$$

Предложение 1 ([8]). Корреляцุионно-иммунная функция порядка $m$ является корреляцุионно-иммунной любого меньшего порядка.

С учетом предложения 1 естественно ввести обозначение

$$
\operatorname{cor} f=\max \{m \in \mathbf{N} \mid f-\text { корреляционно-иммунна порядка } m\}
$$

Следующая теорема является ключевой теоремой, которая используется при исследовании корреляционно-иммунных булевых функций.

Теорема 2 ([8]). Булева функция $f \in \mathscr{F}_{n}$ является корреляционно-иммунной порядка $m$ тогда и только тогда, когда $W_{f}(u)=0$ для всех векторов $u \in V_{n}$ таких, что $1 \leqslant \operatorname{wt}(u) \leqslant m$.

Определение 10. Ортогональной таблицей размера $m \times n$ с ограничениями уровня 2 (в общем случае - уровня $q$, если алфавит имеет мощность $q$ ) силы $t$ и индекса $v$ называется $m \times n$ матрица $M$ над полем $F_{2}$, обладающая следующим свойством: в любом подмножестве из $t$ столбцов матрицы $M$ любой из $2^{t}$ векторов пространства $V_{t}$ встречается как строка ровно $v$ раз. Такая таблица обозначается как $O A_{v}(m, n, 2, t)$.

Для функции $f \in \mathscr{F}_{n}$ будем обозначать через $M_{f}(\operatorname{wt}(f) \times n)$ матрицу, строками которой являются векторы из $V_{n}$, значение функции на которых равно 1 . Мы будем предполагать, что строки выписаны сверху вниз в лексикографическом порядке. Матрицу $M_{f}$ называют таблицей истинности функции $f$.

Теорема 3 ([1]). Для функиии $f \in \mathscr{F}_{n}$ выполнено неравенство соr $f \geqslant t$ тогда $u$ только тогда, когда ее таблийа истинности $M_{f}$ является ортогональной таблицей $O A_{v}(\mathrm{wt}(f), n, 2, t)$.

Определение 11. Введем обозначение

$$
C I(n)=\left\{f \in \mathscr{F}_{n} \mid \operatorname{cor} f \geqslant 1\right\} .
$$

Далее везде под множеством корреляционно-иммунных функций будем понимать множество $C I(n)$. 
Определение 12. Будем называть булеву функцию $f$ от $n$ переменных четной, если для любого вектора $x \in V_{n}$ выполняется равенство $f(x)=f\left(x \oplus 1^{n}\right)$. Обозначим множество всех четных функций через $\operatorname{Mir}(n)$.

Пусть $\mathbb{S}_{V_{n}}-$ симметрическая группа степени $2^{n}$ подстановок на элементах множества $V_{n}$. Рассмотрим некоторую ее подгруппу $c_{5}<\widehat{s}_{V_{n}}$. Отображение

$$
\mathscr{F}_{n} \times(S) \mathscr{F}_{n}, \quad(f, \mathfrak{g}) \rightarrow f^{\mathfrak{g}}, \quad f \in \mathscr{F}_{n}, \quad \mathrm{~g} \in \mathbb{S},
$$

где $f^{\mathfrak{g}}(a)=f(\mathfrak{g} a)\left(\mathfrak{g} a-\right.$ элемент множества $V_{n}$, в который переходит $a$ при действии подстановки g) для любого $a \in V_{n}$, является правым действием группы (s на множестве $\mathscr{F}_{n}$.

Определение 13. Группой инерции функции $f$ в группе (5) называется множество

$$
\tilde{J}_{G}(f)=\left\{g \in \mathbb{G} \mid f^{g}=f\right\} \text {. }
$$

Через $T_{n}$ будем обозначать группу сдвигов, состоящую из подстановок вида

$$
g_{a}: \quad x \rightarrow x \oplus a,
$$

где $x, a \in V_{n}$.

Определение 14. Линейный код $C$ длины $n$ - это линейное подпространство векторного пространства $V_{n}$.

Определение 15. Размерностью $k_{C}$ кода $C$ называется его размерность как векторного пространства $k_{C}=\operatorname{dim} C$.

Определение 16. Минимальным расстоянием $d(C)$ кода $C$ называется минимальное расстояние Хемминга между кодовыми словами

$$
d(C)=\min \left\{\operatorname{dist}\left(c, c^{\prime}\right) \mid c, c^{\prime} \in C, c \neq c^{\prime}\right\} .
$$

Определение 17. Индикаторной функцией множества $S \subseteq V_{n}$ называется такая функция $f=I_{S} \in \mathscr{F}_{n}$, что $f(x)=1$ тогда и только тогда, когда $x \in S$.

Определение 18. Двоичный код Хемминга $H_{r}$ длины $n=2^{r}-1, r>1$, имеет проверочную матрицу $H$, столбцы которой состоят из всех ненулевых двоичных векторов длины $r$, причем каждый вектор встречается в матрице один раз. Код $H_{r}$ имеет параметры

$$
n=2^{r}-1, \quad k=2^{r}-1-r, \quad d=3 .
$$

Двоичным симплексным кодом называется код, который является дуальным к коду Хемминга, то есть код

$$
C^{\perp}=\left\{x \in V_{n} \mid\langle x, c\rangle=0 \text { для всех } c \in C\right\} .
$$

Определение 19. Два двоичных кода называются эквивалентными, если они отличаются только перестановкой координат.

Определение 20. Если $S(n)$ - некоторое подмножество булевых функций от $n$ переменных, то через $S(n, k)$, где $0 \leqslant k \leqslant n$, будем обозначать множество $\{f \in S(n) \mid \operatorname{wt}(f)=k\}$. 
Предложение 2. Если три из четырех булевых функиий $f, g, f \oplus g, f g$ являются корреляционно-иммунными, то и четвертая является корреляционно-иммунной, причем порядок корреляиионной иммунности четвертой функции не меньше минимального из порядков корреляционной иммунности остальных трех функций.

Предложение следует из теоремы 2 и соотношения

$$
F_{f \oplus g}(u)+2 F_{f g}(u)=F_{f}(u)+F_{g}(u),
$$

которое выполнено для любого $u \in V_{n}$ ненулевого веса.

Следствие 1. Если $f, g \in C I(n) u f g \equiv 0$, mо

$$
f \oplus g \in C I(n), \quad \operatorname{cor}(f \oplus g) \geqslant \min (\operatorname{cor} f, \operatorname{cor} g) .
$$

Утверждение непосредственно следует из предложения 2.

Определение 21. Булева функция $f$ от $n$ переменных называется уравновешенной, если $\operatorname{wt}(f)=2^{n-1}$.

Определение 22. Уравновешенная корреляционно-иммунная порядка $d$ функция $f \in \mathscr{F}_{n}$ называется $d$-устойчивой. В дальнейшем под устойчивыми функциями будем понимать уравновешенные функции $f$ такие, что cor $f \geqslant 1$.

\section{3. Некоторые свойства множества четных функций}

Рассмотрим свойства четных функций, которые показывают их тесную связь с множеством корреляционно-иммунных булевых функций.

Предложение 3. Множество $\operatorname{Mir}(n)$ является линейным подпространством пространства $V_{2^{n}}$ размерности $2^{n-1}$.

Предложение следует из определения 12.

Предложение 4. Для любой $f \in \operatorname{Mir}(n)$ справедливь равенства $W_{f}(u)=0$, если $\operatorname{wt}(u)=2 k+1, k \geqslant 0$.

Доказательство. Пусть $f \in \operatorname{Mir}(n)$ и вектор $u \in V_{n}$ таков, что $\operatorname{wt}(u)=2 k+1$ для некоторого натурального числа $k$. Поскольку $1^{n} \in \widetilde{J} T_{n}(f)$, справедливо равенство

$$
f(x)=f\left(x \oplus 1^{n}\right)=f^{\prime}(x)
$$

и для любого $v \in V_{n}$

$$
W_{f}(v)=W_{f^{\prime}}(v)=(-1)^{\left\langle v, 1^{n}\right\rangle} W_{f}(v) .
$$

Для вектора $u$ справедливо равенство $W_{f}(u)=-W_{f}(u)$. Это возможно тогда и только тогда, когда $W_{f}(u)=0$.

Следствие 2. Любая четная функиия является коррелящионно-иммунной как минимум первого порядка.

Доказательство. Для того, чтобы функция $f$ была корреляционно-иммунной как минимум первого порядка, необходимо и достаточно, чтобы $W_{f}(u)=0$ при всех $u$ таких, что $\operatorname{wt}(u)=1$. Из предложения 4 следует, что такое соотношение выполнено для любой четной функции. 
Следствие 3. Для любой неконстантной функции $f \in \operatorname{Mir}(n)$ справедливо соотношение

$$
\operatorname{cor}(f)=2 k+1, \quad k \geqslant 0 .
$$

Доказательство. Если $\operatorname{cor}(f)=n$, то $f-$ константа. Поэтому, из условия следует, что $\operatorname{cor}(f)<n$. Докажем, что $\operatorname{cor}(f)$ не может быть четным числом. Предположим, что существует $f \in \operatorname{Mir}(n)$ такая, что $\operatorname{cor}(f)=2 m$ для некоторого $m \geqslant 1$. Из теоремы 2 следует, что $W_{f}(u)=0$ для всех $u$ таких, что $1 \leqslant \mathrm{wt}(u) \leqslant 2 m$. Из предложения 4 следует, что $W_{f}(u)=0$ при $\operatorname{wt}(u)=2 m+1$. Из теоремы 2 получаем, что $\operatorname{cor}(f)=2 m+1$. Полученное противоречие доказывает утверждение.

Следствие 4. Для множества CI (n) выполнено неравенство

$$
|C I(n)| \geqslant 2^{2^{n-1}} .
$$

Утверждение следует из неравенств

$$
|C I(n)| \geqslant|\operatorname{Mir}(n)|=2^{2^{n-1}} .
$$

Предложение 5. Пусть $f-$ произвольная четная функция. Если для некоторого $i$, $1 \leqslant i \leqslant n$, справедливо неравенство $\operatorname{cor}\left(f_{i}^{0}\right) \geqslant 2 k$, то справедливо неравенство $\operatorname{cor}(f) \geqslant 2 k+1$.

Доказательство. Не ограничивая общности, положим $i=1$. Из предложения 4 следует, что справедливо следующее условие. Функция $f \in \operatorname{Mir}(n)$ является корреляционноиммунной порядка $2 k+1$, если $F_{f}(u)=0$ для любого $u$ такого, что $1 \leqslant \operatorname{wt}(u) \leqslant 2 k$. Для любой четной функции $f$ и для любого набора $u$ четного веса справедливы соотношения

$$
\begin{aligned}
F_{f}(u) & =\sum_{x \in 1_{f}}(-1)^{\langle u, x\rangle} \\
& =\sum_{x \in 1_{f} \& x^{(1)}=0}(-1)^{\langle u, x\rangle}+\sum_{x \in 1_{f} \& x^{(1)}=1}(-1)^{\langle u, x\rangle} \sum_{x \in 1_{f} \& x^{(1)}=0}(-1)^{\langle u, x\rangle}\left(1+(-1)^{\left\langle u, 1^{n}\right\rangle}\right) \\
& =2 F_{f_{1}^{0}}(\tilde{u})
\end{aligned}
$$

где $\tilde{u}=\left(u^{(2)}, \ldots, u^{(n)}\right)$.

Поэтому условие $F_{f}(u)=0$ для любого $u$ такого, что $\operatorname{wt}(u)=2 m$, эквивалентно условию $F_{f_{1}^{0}}(\tilde{u})=0$ для любых $\tilde{u}$ таких, что $2 m-1 \leqslant \operatorname{wt}(\tilde{u}) \leqslant 2 m$. А по условию $\operatorname{cor}\left(f_{1}^{0}\right) \geqslant 2 k$, поэтому $F_{f}(u)=0$ для любых наборов четного веса $w$, где $w \leqslant 2 k$.

Множество $\mathscr{F}_{n}$ булевых функций можно превратить в коммутативное кольцо, определив операцию свертки * булевых функций равенством

$$
(f * g)(x)=\bigoplus_{y \in V_{n}} f(x \oplus y) g(y)=\bigoplus_{z \in V_{n}} f(z) g(z \oplus x)
$$

для любого $x \in V_{n}$. Это кольцо называется групповой алгеброй и обозначается $F_{2} V_{n}$. В общем случае групповая алгебра строится из двух объектов - группы и поля. Некоторые задачи из теории булевых функций удобно рассматривать, пользуясь аппаратом и свойствами групповой алгебры.

При изучении множества $\operatorname{Mir}(n)$ оказалось, что это множество обладает структурными свойствами и относительно операций, введенных в групповой алгебре булевых функций. 
Предложение 6. Множество $\operatorname{Mir}(n)$ является идеалом кольцุа $F_{2} V_{n}$.

Доказательство. Рассмотрим функцию $h=f * g$, где $f \in \operatorname{Mir}(n)$, a $g \in \mathscr{F}_{n}$. Докажем, что для любого набора $x \in V_{n}$ выполняется равенство $h(x)=h\left(x \oplus 1^{n}\right)$. Расписывая $h\left(x \oplus 1^{n}\right)$ по определению операции $*$, получим равенства

$$
h\left(x \oplus 1^{n}\right)=\bigoplus_{y \in V_{n}} f\left(x \oplus y \oplus 1^{n}\right) g(y)=\bigoplus_{y \in V_{n}} f(x \oplus y) g(y) .
$$

Последнее равенство выполнено в силу четности функции $f$. Очевидно также, что $\operatorname{Mir}(n)$ является аддитивной подгруппой кольца $F_{2} V_{n}$. Таким образом, $\operatorname{Mir}(n)$ является идеалом.

\section{4. Структура множества $C I(n)$}

Рассмотрим некоторые структурные свойства множества корреляционно-иммунных булевых функций.

Определение 23. Ядром четности функции $f \in \mathscr{F}_{n}$ назовем функцию $\mathrm{km}(f)$ из $\mathscr{F}_{n}$ вида $g(x)=f(x) f\left(x \oplus 1^{n}\right)$.

Определение 24. Введем обозначение

$$
\operatorname{Anmir}(n)=\left\{f \in \mathscr{F}_{n}, \mathrm{~km}(f) \equiv \overline{0}\right\}
$$

Определение 25. Введем обозначение

$$
B C I(n)=\operatorname{Anmir}(n) \cap C I(n) .
$$

Отметим основные свойства ядра четности корреляционно-иммунной функции.

Предложение 7. Для любой $f \in \mathscr{F}_{n}$ выполняется включение $\operatorname{km}(f) \in \operatorname{Mir}(n)$.

Предложение вытекает из определения 23.

Предложение 8. Для любой $f \in \mathscr{F}_{n}$ выполнено равенство

$$
\operatorname{dist}(f, \operatorname{Mir}(n))=\operatorname{wt}(f \oplus \operatorname{km}(f)) .
$$

Доказательство. Для любой $f \in \mathscr{F}_{n}$ справедливо представление $f=\operatorname{km}(f) \oplus h$, где $h \in \operatorname{Anmir}(n)$ и $h \operatorname{km}(f) \equiv \overline{0}$. Очевидно, что для любой $g \in \operatorname{Mir}(n)$ выполнено неравенство $\operatorname{wt}(g \oplus h) \geqslant \operatorname{wt}(h)$. Также понятно, что существует функция из $\operatorname{Mir}(n)$, для которой это неравенство обращается в равенство, например, $g \equiv \overline{0}$. Поэтому, взяв $g=\operatorname{km}(f)$, получаем, что

$$
\mathrm{wt}(f \oplus g)=\mathrm{wt}(h \oplus k m(f) \oplus g)=\mathrm{wt}(h) .
$$

Далее доказывается утверждение, которое иллюстрирует важность понятия ядра четности для корреляционно-иммунных функций. Из этой теоремы далее будут получены следствия о структуре множества $C I(n)$.

Теорема 4. Для любой функиии $f \in C I(n)$ справедливо включение $(f \oplus \mathrm{km}(f)) \in C I(n)$.

Утверждение теоремы вытекает из предложения 2, предложения 7 и определения 23.

Следствие 5. Для любой $f \in C I(n)$ справедливо равенство $g=f \oplus \operatorname{km}(f), g \in B C I(n)$. 
Утверждение следует из определения $\operatorname{km}(f)$ и множества $B C I(n)$.

Определение 26. Положим

$$
\left.\operatorname{Mir}(n)\right|_{g}=\{f \in \operatorname{Mir}(n) \mid f g \equiv \overline{0}\} .
$$

Предложение 9. Множество $\left.\operatorname{Mir}(n)\right|_{g}$ - линейное подпространство пространства $\operatorname{Mir}(n)$ размерности $2^{n-1}-\operatorname{wt}(g)$.

Предложение следует из определения 26.

В следующем утверждении представлено описание совокупности плоскостей, которые в сумме составляют множество $C I(n)$.

Теорема 5. Для множества CI $(n)$ верно равенство

$$
C I(n)=\bigcup_{g \in B C I(n)}\left(\left.g \oplus \operatorname{Mir}(n)\right|_{g}\right)
$$

Доказательство. Из следствия 5 следует, что любая $f \in C I(n)$ представима в виде $f=g \oplus \mathrm{km}(f)$, где $g \mathrm{~km}(f) \equiv \overline{0}$ и $g \in B C I(n)$. Таким образом, выполнено включение $f \in\left(\left.g \oplus \operatorname{Mir}(n)\right|_{g}\right)$, так как $\operatorname{km}(f) \in \operatorname{Mir}(n)$. Следовательно,

$$
C I(n) \subseteq \bigcup_{g \in B C I(n)}\left(\left.g \oplus \operatorname{Mir}(n)\right|_{g}\right) .
$$

Рассмотрим произвольную функцию $f=g \oplus h$, где $\left.h \in \operatorname{Mir}(n)\right|_{g}$ и $g \in B C I(n)$. Так как для любой функции $h \in \operatorname{Mir}(n)$ справедливо включение $h \in C I(n)$, из предложения 2 следует, что $g \oplus h \in C I(n)$. Следовательно,

$$
\bigcup_{g \in B C I(n)}\left(\left.g \oplus \operatorname{Mir}(n)\right|_{g}\right) \subseteq C I(n) .
$$

Учитывая доказанное выше обратное включение, получаем утверждение теоремы.

Предложение 10. Плоскости, представленные в теореме 5, образуют разбиение множества $C I(n)$.

Доказательство. Предположим обратное, а именно, что существуют $h_{1}, h_{2}$ такие, что $h_{1} \in\left(\left.g_{1} \oplus \operatorname{Mir}(n)\right|_{g_{1}}\right), h_{2} \in\left(\left.g_{2} \oplus \operatorname{Mir}(n)\right|_{g_{2}}\right)$ для $g_{1} \neq g_{2}$ и $h_{1}=h_{2}$. Из определения множества $\left.\operatorname{Mir}(n)\right|_{g}$ видно, что для любой $h \in\left(\left.g \oplus \operatorname{Mir}(n)\right|_{g}\right)$ справедливо равенство $\mathrm{km}(h) \oplus h=g$. Следовательно, $g_{1}=g_{2}$, что противоречит условию и доказывает предложение.

Из данного структурного описания сразу же следует выражение для мощности множества $C I(n)$, которое в свою очередь показывает, что проблема поиска формулы для $|C I(n)|$ сводится к поиску выражения для мощности множеств $B C I(n, w)$ для $w=0,1, \ldots, 2^{n-1}$. Это показывает следующее утверждение.

Следствие 6. Для мощзности множества корреляциионно-иммунных функций как минимум первого порядка справедливо равенство

$$
|C I(n)|=\sum_{g \in B C I(n)} 2^{2^{n-1}-\mathrm{wt}(g)}=\sum_{w=0}^{2^{n-1}} 2^{2^{n-1}-w}|B C I(n, w)| .
$$

Утверждение следует из теоремы 5 и предложения 10. 


\section{5. Нижняя оценка для мощности множества $C I(n)$}

Для функций из множества $\operatorname{Anmir}(n)$ можно предложить специальный способ кодирования, который показывает, что

$$
|\operatorname{Anmir}(n)|=3^{2^{n-1}} .
$$

Приведем описание этого метода. Пусть все наборы $x_{k}$ из $V_{n}$ упорядочены лексикографически. Будем называть вектором значений булевой функции $f \in \mathscr{F}_{n}$ строку $S_{f}$ длины $2^{n}$, элементы которой удовлетворяют соотношениям

$$
S_{f}^{(k)}=f\left(x_{k}\right)
$$

где $S_{f}^{(k)}-k$-й элемент строки, $k=0, \ldots, 2^{n}-1$.

Каждой функции $f \in \operatorname{Anmir}(n)$ взаимно однозначно соответствует некоторая строка $\widetilde{S}_{f}$ длины $2^{n-1}$ с элементами из $F_{3}$. Опишем метод построения этой строки. Пусть $0 \leqslant k \leqslant 2^{n-1}-1$. Элемент $\widetilde{S}_{f}^{(k)}$ равен -1 , если $S_{f}^{(k)}=0$ и $S_{f}^{\left(2^{n}-1-k\right)}=1$. Если $S_{f}^{(k)}=1$ и $S_{f}^{\left(2^{n}-1-k\right)}=0$, то $\widetilde{S}_{f}^{(k)}=1$. Если же $S_{f}^{(k)}=S_{f}^{\left(2^{n}-1-k\right)}=0$, то $\widetilde{S}_{f}^{(k)}=0$. Очевидно, что для $f \in \operatorname{Anmir}(n)$ других вариантов значений на противоположных наборах строки $S_{f}$ нет. По строке $\widetilde{S}_{f}$ однозначно восстанавливается строка $S_{f}$. Таким образом, имеется взаимно однозначное соответствие между функциями из $\operatorname{Anmir}(n)$ и строками, построенными по описанному алгоритму. Таких строк ровно $3^{2^{n-1}}$.

В заключение нужно отметить, что полученные результаты о структуре множества $C I(n)$ позволили улучшить нижнюю оценку мощности этого множества по сравнению с оценкой в следствии 4.

Лемма 1. Для мощности множества $B C I(n, 4 w)$, где $w>1$, выполняется неравенство

$$
|B C I(n, 4 w)| \geqslant\left(\begin{array}{c}
2^{n-2} \\
2 w
\end{array}\right)\left(\begin{array}{c}
2 w \\
w
\end{array}\right) .
$$

Доказательство. Так как $B C I(n)=\operatorname{Anmir}(n) \cap C I(n)$, воспользуемся описанными выше строками $\widetilde{S}_{f}$ для того, чтобы выделить подкласс функций из $\operatorname{Anmir}(n)$ веса $4 w$, которые являются корреляционно-иммунными.

Построим класс $M(n, 4 w) \subset B C I(n)$, который будет состоять из функций $f$, которые удовлетворяют условиям

$$
\begin{gathered}
f_{x^{(1)}}^{0}, f_{x^{(1)}}^{1} \in \operatorname{Mir}(n-1), \quad f_{x^{(1)}}^{0} f_{x^{(1)}}^{1} \equiv 0, \\
\operatorname{wt}\left(f_{x^{(1)}}^{0}\right)=\operatorname{wt}\left(f_{x^{(1)}}^{1}\right)=2 w, \quad w=0,1, \ldots, 2^{n-3} .
\end{gathered}
$$

Разделим строку $\widetilde{S}_{f}$ пополам. В первой половине расставим $2 w$ единиц. Это можно сделать $\left(\begin{array}{c}2^{n-2} \\ 2 w\end{array}\right)$ способами. Из расставленных $2 w$ единиц выберем $w$ штук и заменим их значением -1 . Это можно сделать $\left(\begin{array}{c}2 w \\ w\end{array}\right)$ способами. Оставшиеся позиции в первой половине строки заполним нулями. Заполним вторую половину строки значениями, зеркально отразив относительно середины строки значения первой половины строки $\widetilde{S}_{f}$. Из построения строки $\widetilde{S}_{f}$ следует, что функция $f$ будет удовлетворять всем описанным выше условиям. Понятно, что $f \in B C I(n)$. Получаем, что

$$
B C I(n, 4 w) \geqslant M(n, 4 w)=\left(\begin{array}{c}
2^{n-2} \\
2 w
\end{array}\right)\left(\begin{array}{c}
2 w \\
m
\end{array}\right) .
$$


Теорема 6. Для множества CI(n) справедливо неравенство

$$
|C I(n)| \geqslant \sum_{m=0}^{2^{n-3}} 2^{2^{n-1}-4 m}\left(\begin{array}{c}
2^{n-2} \\
2 m
\end{array}\right)\left(\begin{array}{c}
2 m \\
m
\end{array}\right) .
$$

Утверждение теоремы следует из леммы 1 и следствия 6.

Очевидно, что эта оценка лучше, чем оценка следствия 4. Для примера приведем соотношение между реальным значением $|C I(5)|$ и числами, которые были получены с помощью описанных оценок:

$$
\frac{|C I(5)|}{|M(5)|} \approx 7,6, \quad \frac{|C I(5)|}{|\operatorname{Mir}(5)|} \approx 48 .
$$

\section{6. Минимальность корреляционно-иммунных функций}

Воспользовавшись утверждением предложения 2, можно ввести понятие минимальной корреляционно-иммунной булевой функции.

Определение 27. Функция $f \in C I(n)$ называется минимальной корреляционно-иммунной функцией, если не существует функции $g \in C I(n)$ такой, что $1_{g} \subset 1_{f}$.

Предложение 11. Функция $f \in C I(n)$ является минимальной корреляционно-иммунной функиией тогда и только тогда, когда $f$ нельзя разложить в сумму двух ортогональных корреляционно-иммунных функиий, то есть в сумму двух функиий $g, h \in C I(n)$ таких, что $g h \equiv \overline{0}$.

Предложение вытекает из определения 27.

Учитывая разложение множества $C I(n)$, представленное в теореме 5 , можно сделать следующие выводы о минимальных корреляционно-иммунных функциях малых весов.

Пример 1. Минимальными корреляционно-иммунными булевыми функциями веса 2 являются функции из множества $\operatorname{Mir}(n, 2)$.

Пример 2. Так как для функции $g \in B C I(n, 4)$ не существует $f \in \operatorname{Mir}(n, 2)$ такой, что $1_{f} \subset 1_{g}$, то все функции из $B C I(n, 4)$ являются минимальными веса 4.

Пример 3. Так как для любой функции $f \in B C I(n)$ такой, что $\operatorname{wt}(f)=6$, не существует функции $g \in C I(n)$ такой, что $1_{g} \subset 1_{f}$, то любая функция из $B C I(n, 6)$ является минимальной корреляционно-иммунной функцией веса 6.

Учитывая разложение $C I(n)$ по подпространствам $\left.\operatorname{Mir}(n)\right|_{g}$, приходим к выводу, что множествами $\operatorname{Mir}(n, 2), B C I(n, 4)$ и $B C I(n, 6)$ исчерпываются все минимальные корреляционно-иммунные функции веса 2,4 и 6 . Пользуясь этим же разложением, можно утверждать, что любая минимальная корреляционно-иммунная функция $f$ веса, большего чем 2, принадлежит множеству $B C I(n)$.

Предложение 12. Для любой минимальной корреляциионно-иммунной булевой функции $f$ выполнено неравенство $\operatorname{wt}(f) \leqslant 2^{n-1}$.

Утверждение этого предложения следует из теоремы 5.

Далее оценка веса минимальных корреляционно-иммунных функций будет несколько улучшена. 
Пример 4. Существует 160 минимальных корреляционно-иммунных функций от 5 переменных веса 8. Минимальных функций большего веса от 5 переменных не существует. Минимальная корреляционно-иммунная функция $f$ представлена таблицей истинности. Наборы упорядочены лексикографически, крайнее слева значение соответствует набоpy $1^{n}$.

$$
f=(10010100000000100000001010010100)
$$

Предложение 13. Любая функиия $f \in C I(n)$ может быть разложена в сумму минимальных корреляционно-иммунных булевых функций с попарно непересекающимися носителями.

Доказательство. Пусть $f \in C I(n)$. Если $f$ является минимальной, то утверждение для нее верно. Если $f$ не минимальная, то существует минимальная $f_{1} \in C I(n)$ такая, что $f f_{1}=f_{1}$ и $f=f_{1} \oplus g$, где $g \in C I(n)$. Далее, повторяя для $g$ такие же рассуждения, что и для $f$, получаем разложение функции $f$ на минимальные. Понятно, что носители функций в построенном таким образом разложении не пересекаются.

Разложение функции $f \in C I(n)$ на минимальные не является однозначным.

Пример 5. Функции из $C I(7)$ опишем с помощью задания их носителей:

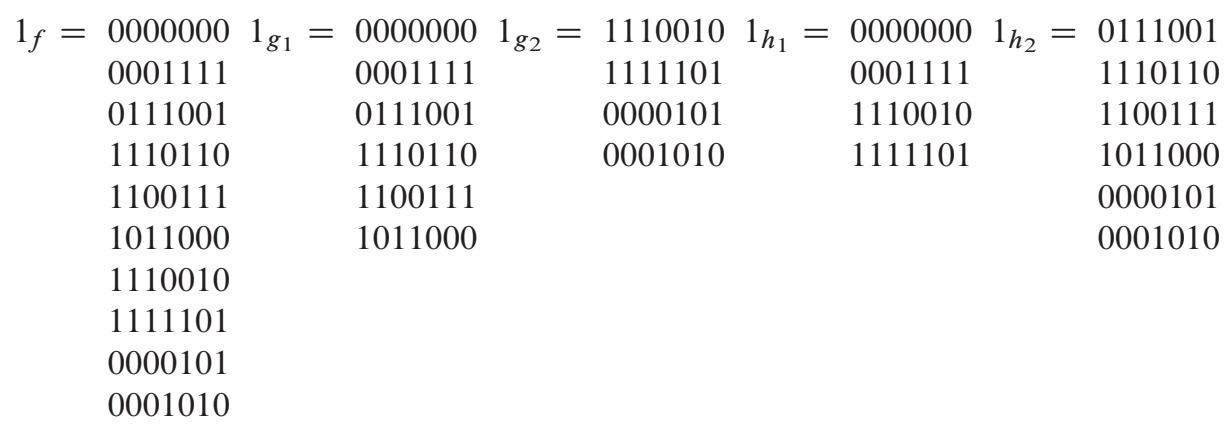

Функция $f$ представима в виде двух сумм $f=g_{1} \oplus g_{2}$ и $f=h_{1} \oplus h_{2}$. Легко видеть, что указанные разложения являются разложениями функции $f$ на минимальные с непересекающимися носителями.

Предложение 14. Для любой минимальной коррелящионно-иммунной булевой функции $f$ от $n$ переменных выполнено соотношение соr $f \leqslant 2$.

Доказательство. Докажем, что для любой булевой функции $f$ такой, что cor $f \geqslant 3$, найдется функция $g \in C I(n)$ такая, что $1_{g} \subset 1_{f}$.

Для таблицы истинности любой функции $f \in \mathscr{F}_{n}$ справедливо представление

$$
f=\left(f_{12}^{11}\left|f_{12}^{10}\right| f_{12}^{01} \mid f_{12}^{00}\right),
$$

где $f_{12}^{b_{1} b_{2}}$ - таблица истинности булевой функции $f_{12}^{b_{1} b_{2}} \in \mathscr{F}_{n-2}$, а знак $\mid$ обозначает конкатенацию. Если cor $f \geqslant 3$, то для любых $b_{1}, b_{2} \in F_{2}$ справедливо неравенство $\operatorname{cor} f_{12}^{b_{1} b_{2}} \geqslant 1$. Поэтому функция $g=\left(f_{12}^{11}|\overline{0}| \overline{0} \mid f_{12}^{00}\right)$ является корреляционноиммунной и $1_{g} \subset 1_{f}$. Следовательно, $f$ не является минимальной корреляционноиммунной функцией. 


\section{7. Минимальные корреляционно-иммунные булевы функции и линейные коды}

В книге [2] рассматривается вопрос построения ортогональных массивов с помощью кодов, исправляющих ошибки. В терминах булевых функций необходимый нам результат можно сформулировать следующим образом.

Теорема 7 ([2]). Если $L<V_{n}$, то cor $I_{L}=r$ тогда и только тогда, когда $d\left(L^{\perp}\right)=r+1$.

Доказательство. Заметим, что функция $S_{u}(x)=\langle u, x\rangle$ либо тождественно равна нулю, либо уравновешена на $L$.

Рассмотрим спектр коэффициентов Фурье функции $f=I_{L}$. Ясно, что

$$
F_{I_{L}}(u)=\sum_{x \in L}(-1)^{\langle u, x\rangle}=\sum_{x \in L}(-1)^{S_{u}(x)}
$$

Предположим, что $d\left(L^{\perp}\right)=r+1$. Тогда существует такой вектор $u \in L^{\perp}$, что $\operatorname{wt}(u)=r+1$. Для этого вектора справедливо неравенство $F_{f}(u) \neq 0$. Для всех $u \in V_{n}$ таких, что $\operatorname{wt}(u) \leqslant r$, функция $S_{u}(x)$ уравновешена. Следовательно, для любого вектора $u \in V_{n}$ такого, что $1 \leqslant \operatorname{wt}(u) \leqslant r$, справедливо равенство $F_{I_{L}}(u)=0$, а значит, cor $I_{L}=r$.

Предположим теперь, что cor $I_{L}=r$. Следовательно, для любого вектора $u \in V_{n}$ такого, что $1 \leqslant \operatorname{wt}(u) \leqslant r$, функция $S_{u}(x)$ уравновешена на $L$. Но существует такой вектор $u \in V_{n}$, что $\operatorname{wt}(u)=r+1$ и $S_{u}(x)=0$ на $L$. Следовательно, $d\left(L^{\perp}\right)=r+1$.

Обозначим через $L C I(n)$ множество корреляционно-иммунных функций от $n$ переменных, которые являются индикаторными функциями множеств $L \oplus z$, где $L<V_{n}$, а $z \in V_{n}$.

Известно, что если $L<V_{n}$, то $\operatorname{deg} I_{L}=n-\operatorname{dim}(L)$.

Определение 28. Функция $f \in L C I(n)$ называется минимальной в классе $L C I(n)$, если не существует такой функции $g \in L C I(n)$, что $1_{g} \subset 1_{f}$.

Предложение 15. Функциия $f=I_{L} \in L C I(n)$ является минимальной в классе LCI $(n)$ тогда и только тогда, когда $L^{\perp}$ нельзя расширить с сохранением свойства $d\left(L^{\perp}\right)>1$.

Доказательство. Докажем необходимость. Если $L^{\perp}<L^{\prime}$, то $L>L^{\prime}$. Если бы $L^{\perp}$ можно было бы расширить с сохранением неравенства $d\left(L^{\perp}\right) \neq 1$, то существовало бы подпространство $L^{\prime}<L$ такое, что $d\left(L^{\prime \perp}\right)>1$. Следовательно, $I_{L^{\prime}} \in L C I(n)$ и $I_{L^{\prime}} I_{L}=I_{L^{\prime}}$. Получили противоречие, которое доказывает необходимость.

Докажем достаточность. Если бы существовала плоскость $L^{\prime}<L$, то выполнялось бы соотношение $L^{\perp}<L^{\prime \perp}$. Если $I_{L^{\prime}} \in C I(n)$, то $d\left(L^{\prime \perp}\right)>1$. Следовательно, можно было бы расширить $L^{\perp}$ с сохранением свойства $d\left(L^{\prime \perp}\right)>1$. Получили противоречие, которое доказывает достаточность.

Предложение 16. Если $L<V_{n}$ такова, что $I_{L}$ является минимальной корреляционноиммунной функиией в классе LCI (n), то выполнено неравенство

$$
\operatorname{dim} L \leqslant \log _{2}(n+1) .
$$

Доказательство. Если $\operatorname{dim} L=m$, то $\operatorname{dim} L^{\perp}=n-m . V_{n}$ состоит из $2^{m}$ различных сдвигов пространства $L^{\perp}$ на векторы из $V_{n}$. Так как $I_{L}$ является минимальной в $L C I(n)$, 
пространство $L^{\perp}$ нельзя расширить так, чтобы для расширенной плоскости $L \frac{\perp}{1}$ coxранялось свойство $d\left(L_{1}^{\perp}\right)>1$. Следовательно, при любом сдвиге пространства $L^{\perp}$ возникает плоскость, в которой присутствуют векторы веса 1. Следовательно, все сдвиги присутствуют в множестве сдвигов на векторы $e_{1}, \ldots, e_{n}$ веса 1 . Поэтому $2^{m} \leqslant n+1$ и $m=\operatorname{dim} L \leqslant \log _{2}(n+1)$.

Предложение 17. Для любой функции $f=I_{L}$, минимальной в классе LCI $(n)$, выполнено неравенство $\operatorname{cor} f \leqslant 2$.

Доказательство. Пусть cor $f \geqslant 3$, тогда $d\left(L^{\perp}\right) \geqslant 4$. Следовательно, $L^{\perp}$ можно расширить с помощью любого вектора веса 2 с сохранением свойства $d\left(L^{\perp}\right)>1$.

Предложение 18. Минимальная корреляциионно-иммунная в классе $L C I(n)$ функцุия $I_{L}$ удовлетворяет условию $\operatorname{cor} I_{L}=2$ тогда и только тогда, когда код $L^{\perp}$ эквивалентен коду Хемминга.

Доказательство. Рассмотрим функцию $f=I_{L}$, которая является минимальной в классе $L C I(n)$. Для нее выполнено неравенство $\operatorname{dim} L \leqslant \log _{2}(n+1)$. Следовательно, $|L|=\operatorname{wt}\left(I_{L}\right)=2^{\operatorname{dim} L} \leqslant n+1$. Если эта функция является корреляционно-иммунной порядка 2, то для нее верно неравенство Рао $\operatorname{wt}(f) \geqslant n+1$. Учитывая предыдущее неравенство, получаем, что минимальная в $L C I(n)$ функция $I_{L}$ может быть корреляционноиммунной порядка 2 в том и только том случае, когда $\operatorname{wt}\left(I_{L}\right)=2^{\operatorname{dim} L}=n+1$. Пусть $\operatorname{dim} L=r$. Тогда пространство $L^{\perp}$ имеет параметры $n=2^{r}-1, \operatorname{dim} L^{\perp}=2^{r}-1-r$, $d\left(L^{\perp}\right)=3$. Но любой линейный код с такими параметрами эквивалентен коду Хемминга.

Таким образом, для всех функций $f=I_{L} \in L C I(n)$ таких, что cor $f=2$, являющихся минимальными в этом классе, код $L^{\perp}$ эквивалентен коду Хемминга.

Пример 6. Существуют минимальные корреляционно-иммунные булевы функции порядка 2. Функцию $f \in C I(7)$ опишем с помощью задания ее носителя

$$
\begin{array}{r}
1_{f}=0000000 \\
0001111 \\
0110011 \\
0111100 \\
1010101 \\
1011010 \\
1100110 \\
1101001
\end{array}
$$

Носитель функции $f$ является двоичным симплексным кодом.

Вернемся к вопросу оценки веса минимальных корреляционно-иммунных булевых функций.

Лемма 2. Для любой уравновешенной булевой функции $f \in B C I(n)$ выполняется равенство $\operatorname{cor} f=2 k, k \in \mathbf{N}$.

Доказательство. Пусть $f \in B C I(n)$ и $\operatorname{wt}(f)=2^{n-1}$. Рассмотрим вектор $u \in V_{n}$ такой, что $\operatorname{wt}(u)=2 k$ для $k=1, \ldots,\lfloor n / 2\rfloor$. Ясно, что

$$
W_{f}(u)=\sum_{x \in V_{n}}(-1)^{f(x) \oplus\langle u, x\rangle}=\sum_{x \in V_{n}, x^{(1)}=0}(-1)^{f(x) \oplus\langle u, x\rangle}+(-1)^{f\left(x \oplus 1^{n}\right) \oplus\left\langle u, x \oplus 1^{n}\right\rangle}=0 .
$$

Следовательно, существует число $k \in \mathbf{N}$ такое, что $k \leqslant\lfloor n / 2\rfloor$ и cor $f=2 k$. 
Пример 7. Существуют устойчивые булевы функции от трех переменных, которые являются минимальными. Одна из функций представлена таблицей истинности $f=(10010110)$.

Теорема 8. Для произвольной устойчивой булевой функции от $n \geqslant 4$ переменных порядка $k \geqslant 1$ существуют подпространство $L<V_{n}$ и вектор $z \in V_{n}$ такие, что $I_{L \oplus z} \in C I(n)$ $u L \oplus z \subseteq 1_{f}$.

Доказательство. Если существует вектор $x \in V_{n}$ такой, что $f(x)=f\left(x \oplus 1^{n}\right)$, то искомым пространством является $L=\left\{0^{n}, 1^{n}\right\}$, а $z=x$.

Если $f \in B C I(n)$, то по лемме $2 \operatorname{cor} f=2 k$ для некоторого $k \in \mathbf{N}$ и $k \leqslant\lfloor n / 2\rfloor$. Рассмотрим подфункцию $f_{x^{(1)}}^{0} \in \mathscr{F}_{n-1}$. Порядок ее корреляционной иммунности не меньше $2 k-1$.

Предположим, что $f_{x^{(1)}}^{0}$ не принадлежит множеству $B C I(n-1)$, тогда существует такой вектор $z_{0} \in V_{n-1}$, что $f_{x^{(1)}}^{0}\left(z_{0} \oplus 1^{n-1}\right)=f_{x^{(1)}}^{0}\left(z_{0}\right)=1$. Тогда для функции $f_{x^{(1)}}^{1}$ существует вектор $z_{1} \in V_{n-1}$, удовлетворяющий аналогичным условиям. Пусть $\tilde{z}_{0}$ и $\tilde{z}_{1}$ - векторы из $V_{n}$, полученные, соответственно, из векторов $z_{0}$ присоединением нулевой первой компоненты и из $z_{1}$ присоединением единичной первой компоненты. Пусть $\tilde{y}=(01 \ldots 1)$ - вектор из $V_{n}$. Тогда искомая плоскость определяется следующим образом: $L=\left\{\tilde{z}_{0}, \tilde{z}_{1}, \tilde{z}_{0} \oplus \tilde{y}, \tilde{z}_{1} \oplus \tilde{y}\right\}$.

Если $f_{x^{(1)}}^{0} \in B C I(n-1)$, то $f_{x^{(1)}}^{0}=f_{x^{(1)}}^{1}$. Следовательно, переменная $x^{(1)}$ является фиктивной для функции $f$. Справедливость утверждения теоремы для функции $f_{x(1)}^{0} \in \mathscr{F}_{n-1}$ влечет справедливость утверждения для функции $f \in \mathscr{F}_{n}$. Таким образом, применяя приведенные выше рассуждения, мы либо получим искомую плоскость для функции $f_{x^{(1)}}^{0}$, либо перейдем к рассмотрению функции от меньшего числа переменных. Для функций от $n=4$ переменных существуют лишь две устойчивые функции из $B C I(n)$, у которых подфункции также принадлежат множеству $B C I(n)$, а именно, $f_{1}=(0110100101101001)$, $f_{2}=(100101101001$ 0110). Функциями, которые показывают справедливость утверждения теоремы для функций $f_{1}$ и $f_{2}$, являются функции $g_{1}=(0110000000001001)$, $g_{2}=(1001000000000110)$.

Следствие 7. Для любых натурального $n \geqslant 4$ и булевой функиии $f \in M C I(n)$ справедливо неравенство $\operatorname{wt}(f)<2^{n-1}$.

Утверждение следует из предложения 12 и теоремы 8.

\section{8. Корреляционно-иммунные функции веса 4}

Рассмотрим некоторые свойства корреляционно-иммунных функций веса 4. С использованием этих свойств удалось получить выражение для мощности множества таких функций.

Предложение 19. Любая функция $f \in B C I(n, 4)$ представима в виде $f=I_{L \oplus z}$ для некоторого подпространства $L<V_{n}$ такого, что $\operatorname{dim} L=2$, и вектора $z \in V_{n}$.

Доказательство. Для любой функции $f \in B C I(n, 4)$ и для любого вектора $z \in 1_{f}$ выполнено следующее условие: функция $f^{\prime}$ такая, что $f^{\prime}(x)=f(x \oplus z)$, принадлежит множеству $B C I(n, 4)$. Поэтому, не ограничивая общности, рассмотрим множество $L=\left\{0^{n}, x_{1}, x_{2}, x_{3}\right\}$ такое, что $f=I_{L} \in B C I(n, 4)$. 
Так как $f \in B C I(n)$, справедливо соотношение $x_{i} \oplus x_{j} \neq 1^{n}$. Рассмотрим $x_{1}, x_{2}$, $x_{3}$ и $0^{n}=x_{4}$. Если $x_{1}^{(j)}=0$, то $x_{2}^{(j)}$ и $x_{3}^{(j)}=1$. Если $x_{1}^{(j)}=1$, то $x_{2}^{(j)} \oplus x_{3}^{(j)}=1$. Следовательно, $x_{2} \oplus x_{3}=x_{1}$ и $L$ является линейным подпространством пространства $V_{n}$.

Следовательно, для любой функции $f \in B C I(n, 4)$ существует подпространство $L<V_{n}$ и вектор $z \in V_{n}$ такие, что $f=I_{L \oplus z}$.

Пространство $L<V_{n}$, которое является носителем функции $f \in B C I(n, 4)$, обладает следующими свойствами: $|L|=4$ и для любых $x, y \in L$ справедливы соотношения $x \oplus y \neq 1^{n}$ и $x_{1} \vee x_{2} \vee x_{3} \vee x_{4}=1^{n}$.

Предложение 20. Справедливо равенство $|B C I(n, 4)|=2^{n-3}\left(3^{n-1}-2^{n}+1\right)$.

Доказательство. Пусть $L<V_{n}$ и $L=\left\{x_{0}=0^{n}, x_{1}, x_{2}, x_{3}\right\}$. Не ограничивая общности, положим $x_{1}^{(1)}=0, x_{2}^{(1)}=1$ и $x_{3}^{(1)}=0$.

Обозначим $\tilde{x}_{i}$ строку $x_{i}$ без первого значения $x_{i}^{(1)}$. В строке $\tilde{x}_{1}$ может быть $k$ нулей при $0 \leqslant k \leqslant n-2$. Фиксировав строку $\tilde{x}_{1}$, мы получим ограничения на строки $\tilde{x}_{2}$ и $\tilde{x}_{3}$. $\mathrm{B}$ тех позициях, в которых в строке $\tilde{x}_{1}$ стоят нули, должны стоять значения 1 в $\tilde{x}_{2}$ и $\tilde{x}_{3}$. В тех позициях, в которых в $\tilde{x}_{1}$ стоят единицы, должны стоять противоположные значения в строках $\tilde{x}_{2}$ и $\tilde{x}_{3}$. Таким образом, после фиксации значения $\tilde{x}_{1}$ однозначно определяются значения в $k$ позициях строк $\tilde{x}_{2}$ и $\tilde{x}_{3}$. Понятно, что та часть строки $\tilde{x}_{2}$, которая осталась нефиксированной, однозначно определяет значение той части строки, которая осталась нефиксированной в $\tilde{x}_{3}$. Если выделить нефиксированную часть строк $\tilde{x}_{2}$ и $\tilde{x}_{3}$, то получим векторы $X_{2}$ и $X_{3}$ такие, что $X_{2}=X_{3} \oplus 1^{n-1-k}$. Векторы $X_{2}, X_{3} \in V_{n-1-k}$. Вектор $X_{2}$ не может принимать значения $0^{n-1-k}, 1^{n-1-k} \in V_{n-1-k}$. Так как порядок столбцов $X_{2}$ и $X_{3}$ неважен, число разных $X_{2}$ и $X_{3}$ при фиксированном $\tilde{x}_{1}$ с $k$ нулями равно $\left(2^{n-k-1}-2\right) / 2=2^{n-k-2}-1$.

Таким образом, мы получили соотношения

$$
\begin{aligned}
\left|\left\{I_{L} \in B C I(n, 4) \mid L<V_{n}\right\}\right| & =\sum_{k=0}^{n-2}\left(\begin{array}{c}
n-1 \\
k
\end{array}\right) 2^{n-k-2}-\sum_{k=0}^{n-2}\left(\begin{array}{c}
n-1 \\
k
\end{array}\right) \\
& =\frac{1}{2}\left(\sum_{k=0}^{n-1}\left(\begin{array}{c}
n-1 \\
k
\end{array}\right) 2^{n-1-k}-1\right)-2^{n-1}+1 \\
& =\frac{1}{2}\left(3^{n-1}-1\right)-2^{n-1}+1=\frac{1}{2}\left(3^{n-1}-2^{n}+1\right) .
\end{aligned}
$$

Для того, чтобы посчитать $|B C I(n, 4)|$, нужно также учесть функции, которые получаются из подпространств путем сдвига на некоторые векторы. Для подпространств $L_{i} \neq L_{j}$ и любых векторов $x, y \in V_{n}$ справедливо соотношение $L_{i} \oplus x \neq L_{j} \oplus y$, поэтому $|B C I(n, 4)|=2^{n-2}\left|\left\{I_{L} \in B C I(n, 4) \mid L<V_{n}\right\}\right|=2^{n-3}\left(3^{n-1}-2^{n}+1\right)$, что и требовалось доказать.

Следствие 8. Справедливо равенство $|\{f \in C I(n) \mid \operatorname{wt}(f)=4\}|=2^{n-3}\left(3^{n-1}-1\right)$.

Утверждение следует из предложения 20 и равенства $C I(n)=\bigcup_{g \in B C I(n)}\left(\left.g \oplus \operatorname{Mir}(n)\right|_{g}\right)$.

\section{9. Пространства корреляционно-иммунных функций}

Пусть $L<V_{n}$ таково, что $I_{L} \in C I(n)$. Рассмотрим всевозможные различные сдвиги $L$ на $z \in V_{n}$. Все пространство $V_{n}$ состоит из $2^{n-\operatorname{dim} L}$ различных сдвигов пространства $L$ на векторы из $V_{n}$. 
Пусть $P_{L}=\left\{L, L \oplus z_{1}, \ldots, L \oplus z_{2^{n-\operatorname{dim} L-1}}\right\}$. Если $P_{L}^{i}=L \oplus z_{i}$, то для всех $i, j, i \neq j$, выполнено $P_{L}^{i} \cap P_{L}^{j}=\varnothing$ и $I_{P_{L}^{i}} \in C I(n)$. Следовательно, $Z\left(\left\{I_{P_{L}^{i}}\right\}_{i=0}^{2^{n-\operatorname{dim} L}-1}\right) \subseteq C I(n)$, где $Z(S)$ означает линейную оболочку, натянутую на векторы из множества $S$.

Пусть $T_{n}$ - множество сдвигов на векторы из $V_{n}, J_{T_{n}}(f)$ - группа инерции функции $f$ в группе $T_{n}$.

Предложение 21. Пусть $f \in \mathscr{F}_{n}$. Если $J_{T_{n}}(f) \geqslant L$, то функиия $f$ представима в виде

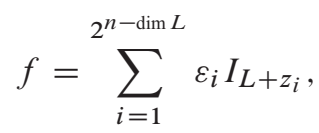

где $\varepsilon_{i} \in\{0,1\}, z_{i} \in V_{n}$, mo есть $f \in Z\left(\left\{P_{L}^{i}\right\}_{i=0}^{2^{n-\operatorname{dim} L}-1}\right)$.

Доказательство. Из условия предложения видно, что $1_{f} \oplus v=1_{f}$ для любого $v \in L$. Таким образом, для любого $z \in 1_{f}$ и любого $v \in L$ существует такой вектор $y \in 1_{f}$, что $z \oplus v=y$. Рассмотрим произвольный вектор $z_{1} \in 1_{f}$. Справедливо включение $L \oplus z_{1} \subseteq 1_{f}$. Рассмотрим произвольный вектор $z_{2} \in 1_{f} \backslash\left(L \oplus z_{1}\right)$. Справедливо включение $L \oplus z_{2} \subseteq 1_{f} \backslash\left(L \oplus z_{1}\right)$. Продолжая далее рассмотрение векторов $z_{i}$, определяемых по описанной схеме, получим, что множество $1_{f}$ исчерпывается некоторым числом сдвигов плоскости $L$.

Признаком принадлежности произвольной булевой функции классу $L C I(n)$ может служить свойство существования такого подпространства $L<V_{n}$, что $L \leqslant J_{T_{n}}(f)$ и $d\left(L^{\perp}\right)>1$.

Введем обозначение $Z\left(\left\{P_{L}^{i}\right\}_{i=0}^{2^{n-\operatorname{dim} L}-1}\right)=\mathscr{L}(L)$.

Понятно, что $|\mathscr{L}(L)|=2^{2^{n-\operatorname{dim} L}}$, причем, если $L<V_{n}$ таково, что $I_{L}-$ минимальная корреляционно-иммунная функция в классе $L C I(n)$, то нельзя указать другое подпространство $S<V_{n}$ такое, что $\mathscr{L}(S) \supset \mathscr{L}(L)$.

Свойства пространства $\mathscr{L}(L)$ с точки зрения алгебраической вырожденности функций были рассмотрены в [11]. В терминах данной работы можно сформулировать несколько более общие результаты.

В дальнейшем будем отождествлять подстановки $g_{a} \in T_{n}$ и векторы $a \in V_{n}$ в силу изоморфизма групп $T_{n} \cong V_{n}$. Пусть $J_{T_{n}}(f)-$ группа инерции функции $f \in \mathscr{F}_{n}$ в группе $T_{n}$.

Будем говорить, что $\operatorname{cor}(f)=0$, если функция $f$ не является корреляционно-иммунной хотя бы первого порядка.

Предложение 22. Пусть $f \in \mathscr{F}_{n} u \operatorname{cor}(f)=k, k \geqslant 0$. Пусть $S=\left\{z_{1}, \ldots, z_{m}\right\} \subseteq V_{n}$ таково, что $\left(1_{f} \oplus z_{i}\right) \cap\left(1_{f} \oplus z_{j}\right)=\varnothing$ при $i \neq j$. Если для любого вектора $u \in V_{n}$ такого, что $\operatorname{wt}(u)=k+1$, выполняется соотношение $F_{I_{S}}(u) F_{f}(u)=0$, то для функции $g=\bigoplus_{i=1}^{m} I_{1_{f} \oplus z_{i}}$ справедливо неравенство $\operatorname{cor}(g) \geqslant k+1$.

Доказательство. Для любого вектора $u \in V_{n}$ рассмотрим коэффициент Фурье $F_{g}(u)$ функции $g$ :

$$
\begin{aligned}
F_{g}(u) & =\sum_{x \in 1_{g}}(-1)^{\langle u, x\rangle}=\sum_{i=1}^{m} \sum_{x \in 1_{f} \oplus z_{i}}(-1)^{\langle u, x\rangle} \\
& =\sum_{i=1}^{m} \sum_{x \in 1_{f}}(-1)^{\langle u, x\rangle}(-1)^{\left\langle u, z_{i}\right\rangle}=\sum_{i=1}^{m}(-1)^{\left\langle u, z_{i}\right\rangle} \sum_{x \in 1_{f}}(-1)^{\langle u, x\rangle}=F_{I_{S}}(u) F_{f}(u) .
\end{aligned}
$$


Очевидно, что $\operatorname{cor}(g) \geqslant \operatorname{cor}(f)$. Далее, так как по условию для любого вектора $u \in V_{n}$ такого, что $\operatorname{wt}(u)=k+1$, выполнено равенство $F_{I_{S}}(u) F_{f}(u)=0$, для этого вектора равенство $F_{g}(u)=0$ также справедливо. Следовательно, $\operatorname{cor}(g) \geqslant \operatorname{cor}(f)+1$.

Справедливо более общее утверждение.

Предложение 23. Пусть $f \in \mathscr{F}_{n} u \operatorname{cor}(f)=k, k \geqslant 0$. Пусть $S=\left\{z_{1}, \ldots, z_{m}\right\} \subseteq V_{n}$ таково, что $\left(1_{f} \oplus z_{i}\right) \cap\left(1_{f} \oplus z_{j}\right)=\varnothing$ при $i \neq j$. Пусть $t_{0}-$ максимальное значение, для которого для всех $u \in V_{n}$ таких, что $\operatorname{wt}(u)=t_{0}$, справедливо равенство $F_{I_{S}}(u) F_{f}(u)=0$. Тогда для функции $g=\bigoplus_{i=1}^{m} I_{1_{f} \oplus z_{i}}$ выполняется равенство $\operatorname{cor}(g)=\max \left\{t_{0}, k\right\}$.

Доказательство аналогично доказательству предыдущего предложения.

Заметим, что пространство $\mathscr{L}(L)$, где $I_{L} \in C I(n)$, может быть вложено в некоторое большее пространство, которое тоже состоит из корреляционно-иммунных функций.

Предложение 24. Пусть подпространство $L<V_{n}$ таково, что $I_{L} \in C I(n) u$ $\left\{z_{0}=0^{n}, z_{1}, \ldots, z_{2^{n-\operatorname{dim}} L_{-1}}\right\}$ - векторы из попарно различных классов смежности по подпространству $L$. Если $I_{L}=I_{M_{1}} \oplus \ldots \oplus I_{M_{k}}-$ разложение функиии $I_{L}$ на минимальные корреляциионо-иммунные функции, то $\mathscr{L}(L) \subseteq \mathscr{L}\left(M_{1}, \ldots, M_{k}\right) \subset C I(n)$, где $\mathscr{L}\left(M_{1}, \ldots, M_{k}\right)$ - линейная оболочка, натянутая на функции из множества $M=\left\{I_{M_{1} \oplus z_{0}}, \ldots, I_{M_{1} \oplus z_{2^{n-\operatorname{dim} L}-1}}, \ldots, I_{M_{k} \oplus z_{0}}, \ldots, I_{M_{k} \oplus z_{2^{n-\operatorname{dim} L-1}}}\right\}$.

Доказательство следует из того, что функции из множества $\mathcal{M}$ имеют попарно непересекающиеся носители и являются корреляционно-иммунными функциями.

\section{Список литературы}

1. Логачев О. А., Сальников А. А., Ященко В. В., Булевы функиии в теории кодирования и криптологии. МЦНМО, Москва, 2004.

2. Hedayat A. S., Sloane N. J. A., Stufken J., Orthogonal arrays. Theory and applications. Springer, Berlin, 1999.

3. Pott A., Group algebras and correlation immune functions. Lecture Notes Computer Sci. (2005) 3486, 437-450.

4. Maitra S., Sarkar P., Enumeration of correlation immune Boolean functions. Lecture Notes Computer Sci. (1999) 1587, 12-25.

5. Maitra S., Boolean functions with important cryptographic properties, $\mathrm{PhD}$ Thesis, Indian Statist. Inst., 2001.

6. Таранников Ю. В., О корреляционно-иммунных и устойчивых булевых функциях. Матем. вопросы кибернетики (2002) 11, 91-148.

7. Siegenthaler T., Decrypting a class of stream ciphers using ciphertext only. IEEE Trans. Computers (1985) 34, 81-85.

8. Siegenthaler T., Correlation-immunity of nonlinear combining functions for cryptographic applications. IEEE Trans. Inform. Theory (1984) 30, 776-780.

9. Maitra S., Autocorrelation properties of correlation immune Boolean functions. Lecture Notes Computer Sci. (2001) 2247, 242-253.

10. Ботев А. А., О свойствах корреляционно-иммунных функций с высокой нелинейностью, Дисс. на соискание ученой степени кандидата физ.-матем. наук, Москва, 2005.

11. Dawson E., Wu C. K., Construction of correlation immune Boolean functions. Lecture Notes Computer Sci. (1997) 1334, 170-180.

Статья поступила 21.01.2009. 